\title{
REPOSITORIOS DE RECURSOS EDUCATIVOS ABIERTOS
}

\author{
Javiera Atenas-Rivera, Francisco Rojas-Sateler y Mario Pérez-Montoro
}
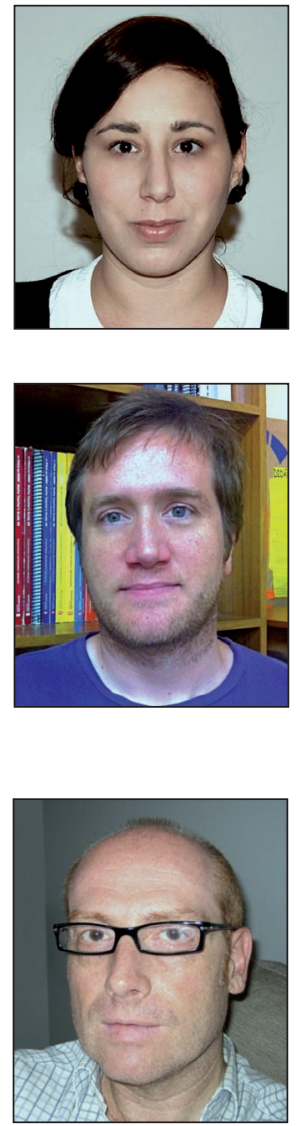

Javiera Atenas-Rivera, doctoranda de la Universitat de Barcelona (UB), trabaja en innovación tecnológica en el Depto. de Desarollo Académico en la escuela de estudios africanos y orientales de la University of London, Inglaterra. Es lectora en el curso de nuevas tecnologías y medios sociales para museos de IL3 de la UB y tiene un máster en gestión del conocimiento por la Hoschshule Hamburg (Alemania). Investiga en nuevas tecnologías para la educación. Es voluntaria de la Open Knowledge Foundation.

SOAS, University of London Thornhaugh Street, Russell Square, London WC1H OXG javiera.atenas@gmail.com

Francisco Rojas-Sateler es doctor por la Universitat Autònoma de Barcelona (UAB) en didáctica de las matemáticas y las ciencias experimentales. Trabaja en el Centro Félix Klein de la Universidad de Santiago de Chile de investigación y transferencia en didáctica de las matemáticas, donde es coordinador editorial y está a cargo de la adaptación para Chile de textos escolares internacionales. Sus líneas de investigación son el estudio de la comunicación y discurso en el aula de matemáticas desde enfoques competenciales y epistemológicos.

Centro Félix Klein Universidad de Santiago de Chile Av. Schatchtebeck, 4. Santiago, Chile franciscorojas.klein@gmail.com

Mario Pérez-Montoro es doctor en filosofía y ciencias de la educación por la Universitat de Barcelona $(U B)$ y máster en organización de sistemas de documentación en la empresa por la Universitat Politècnica de Catalunya (UPC). Ha realizado estudios de postgrado en la Università di Bologna (Italia) y ha sido profesor visitante en la Stanford University (California, EUA) y en la UC Berkeley (California, EUA). Su trayectoria científica se centra sobre algunos aspectos (conceptuales, semánticos, epistemológicos y pragmáticos) relacionados con la teoría de la información y la gestión del conocimiento.

Universitat de Barcelona Facultat de Biblioteconomia y Documentació Melchor de Palau, 140. 08014 Barcelona perez-montoro@ub.edu

\section{Resumen}

Los repositorios de recursos educativos abiertos de aprendizaje facilitan el acceso al conocimiento, proporcionando oportunidades para que las instituciones de educación superior compartan materiales didácticos que pueden mejorar el aprendizaje. Los materiales pueden reutilizarse, traducirse y modificarse, y compartirse incluso a través de redes sociales, de modo que son accesibles desde cualquier lugar, sin barreras económicas o de derechos de autor. Permiten la democratización de la enseñanza.

\section{Palabras clave}

Conocimiento, Recursos educativos abiertos, Acceso abierto, Educación democrática, Repositorios.

\section{Title: Open educational resources repositories as academic information tools}

\section{Abstract}

The open educational resources (OER) repositories facilitate access to knowledge, creating opportunities for academic institutions to share pedagogical materials that can enhance the teaching and learning experience in higher education. They can be reused, translated or modified, and even disseminated through social networks, making these multimedia materials accessible anywhere at any time, without economic and copyright barriers, thereby encouraging active participation in the democratic education movement.

\section{Keywords}

Knowledge, Open educational resources, Open access, Democratic education, Repositories. 
Atenas-Rivera, Javiera; Rojas-Sateler, Francisco; Pérez-Montoro, Mario. "Repositorios de recursos educativos abiertos como herramientas de información académica". El profesional de la información, 2012, marzo-abril, v. 21, n. 2, pp. 190-193.

\section{Introducción}

Los recursos educativos abiertos [open educational resources (OER)] fueron mencionados por primera vez en 2002 en una conferencia de la Unesco $(2002$, p. 16) definiéndose como: "recursos educativos basados en las tecnologías de la información y la comunicación, para su consulta, uso y adaptación por parte de una comunidad de usuarios con fines no comerciales". La OECD (2007, p. 11) los define como "materiales digitalizados ofrecidos libre y abiertamente a profesores, alumnos y autodidactas para que sean usados y reutilizados para enseñar, aprender e investigar". Posteriormente la Unesco $(2011$, p. 5) los definió como "recursos de aprendizaje que incluyen [...] materiales didácticos interactivos, libros digitales, vídeos, aplicaciones multimedia, podcasts y otros diseñados con fines educativos, que pueden ser compartidos en red, puestos a disposición de los docentes, académicos y estudiantes, y accesibles sin necesidad de pagar por suscripciones o licencias".

Mientras los recursos educativos abiertos son criticados por Gurell, Kuo y Walker (2010) por considerarlos una solución técnica o de ingeniería a un problema fundamentalmente pedagógico, McGreal (2004) tiene en cuenta su valor pedagógico y de difusión del conocimiento abierto.

A los problemas de la crisis económica iniciada en 2008, con los consiguientes recortes presupuestarios a la educación en muchos países, en el mundo académico se ha unido el descontento por los precios que la industria editorial aplica a los artículos, libros y materiales didácticos que publica. Su elevado coste ha hecho que las universidades opten por modelos de acceso abierto.

En este contexto, los repositorios universitarios -ya sean de documentos o de materiales didácticos- juegan un rol importante, ya que generalmente son de libre acceso (o al menos tienen políticas menos restrictivas), y los costes de su gestión son asumibles y sustentables. Algunos incluyen opciones como el acceso desde dispositivos móviles o la difusión a través de redes sociales, lo cual aumenta la posibilidad de poder compartir los materiales que tienen archivados.

El mayor beneficio de los repositorios, tanto para investigadores y profesorado como para estudiantes, es el acceso permanente a sus colecciones y recursos de aprendizaje de manera estructurada (Carson, 2005). En general, son los mismos académicos, bibliotecarios y diseñadores de contenidos quienes elaboran tales recursos.

\section{Flexibilidad de los OERs}

Al diseñarlos se deben considerar ciertas características técnicas que faciliten su portabilidad global. Así, por ejemplo, si se trata de un programa informático su código fuente debe ser público, y no se deben interponer barreras económicas, como suscripciones o delimitaciones de copyright. Los docentes deben ser capaces no sólo de usar los recursos, sino también de adaptarlos, construir sobre ellos, volverlos a utilizar y compartirlos con otros docentes. Por todo ello es aconsejable usar licencias Creative commons (CC) con reconocimiento del autor (BY), uso no comercial (NC), y compartir igual o share alike (SA), o sea CC-BY-NC-SA.

La Open Knowledge Foundation define los contenidos abiertos como "los que cualquier persona es libre de utilizar, reutilizar, y redistribuir, siendo su única responsabilidad atribuirlo y compartirlo por igual". Según Downes (2007), el concepto de abierto implica "coste cero" para el usuario. Para Daniel (2010) sin embargo, más que enfocados en el coste, los recursos deben ser accesibles, adecuados, asequibles y acreditados, y para ello son adecuadas las licencias Creative commons.

\section{Calidad y requerimientos}

La creación de ROERs, según Siemens (2003), es importante para preservar la educación pública, ya que los recortes presupuestarios amenazan potencialmente el sistema público de educación. El libre intercambio de recursos educativos de calidad puede asegurar la permanencia de los ideales democráticos de la educación pública, alcanzando estándares educativos altos que puedan competir con el modelo industrializado de la educación privada.

El profesorado comparte la información de diferentes formas, y actualmente está en auge hacerlo a través de las redes sociales. Algunos repositorios como Xpert de la University of Nottingham y OER Commons facilitan enlaces para compartir recursos en redes como Twitter o Facebook entre otras.

http://www.nottingham.ac.uk/xpert

http://www.oercommons.org

En internet hay millones de documentos y materiales multimedia que pueden ser encontrados mediante motores de búsqueda, pero es difícil garantizar que la información que se recupera sea adecuada en un ámbito pedagógico. Por ello, los repositorios institucionales indexados mediante metadatos facilitan la búsqueda de información por parte de los docentes, al mismo tiempo que garantizan la calidad de los materiales.

Para Longmire (2000), el reto mayor al que se enfrentan quienes elaboran repositorios es la implementación de sistemas de recuperación que permitan a los docentes encontrar los contenidos de aprendizaje apropiados a sus necesidades. Según Margaryan et al. (2007), quienes desarrollan ROERs deben ser capaces de determinar con exactitud los usuarios finales de los recursos y las metas pedagógicas de las personas que los utilizan, considerando las necesidades y las características culturales y socioeconómicas de las comunidades de usuarios actuales y potenciales. 


\begin{tabular}{|c|c|}
\hline Back to main page & 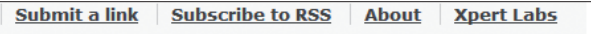 \\
\hline $\begin{array}{l}\text { Currently searching } 253936 \text { resources. } \\
\text { Please enter your keywords }\end{array}$ & $\begin{array}{l}\text { Popular Tags } \\
\text { Education Science Podcasts Humanities } \\
\text { Politics Engineering Organic.Edunet Web Portal } \\
\text { Literature History Business mathematics Arts art } \\
\text { knowledge wharton museum Religion English } \\
\text { politics news General education media society Art } \\
\text { Klartext Podradio SR P4 TV }\end{array}$ \\
\hline
\end{tabular}

http://www.nottingham.ac.uk/xpert

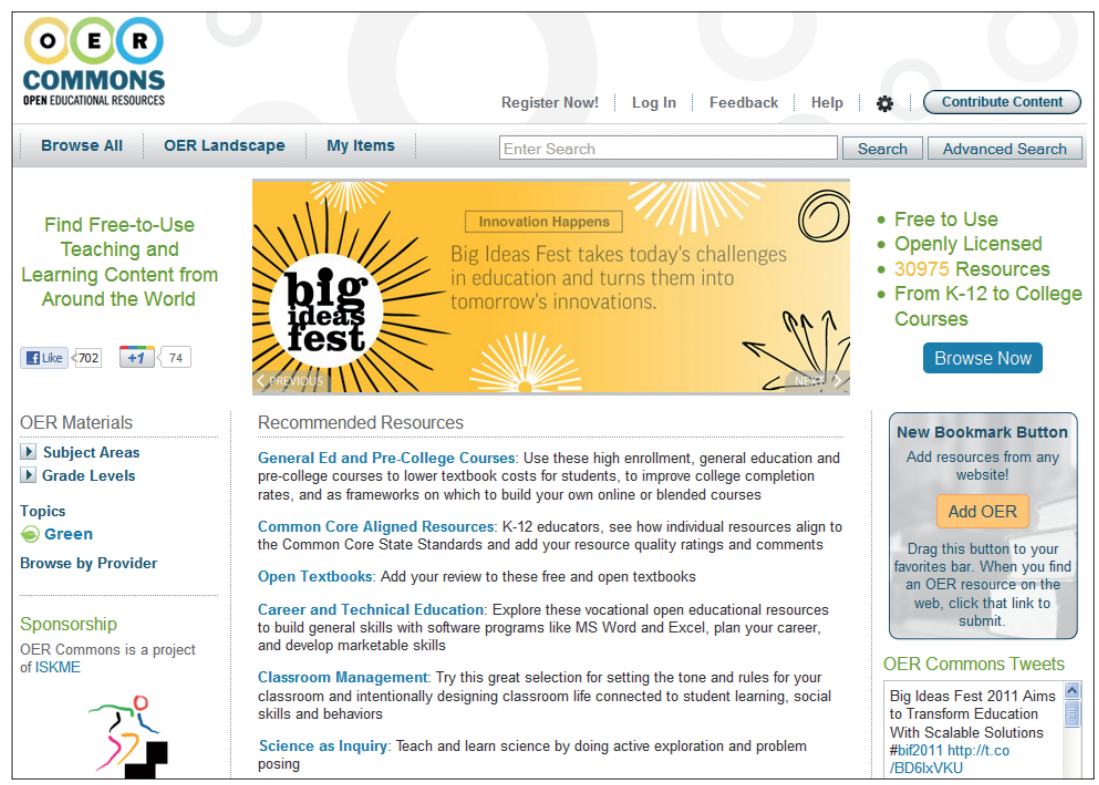

http://www.oercommons.org

\section{Importancia de la utilización de metadatos}

Para Rodríguez-Morales y Cueva-Carrión (2010) es crucial la correcta indexación y una buena usabilidad que faciliten el acceso a los materiales educativos. Además es necesario el desarrollo de estándares y modelos de gestión de la información con esquemas definidos de metadatos que faciliten la interoperabilidad de los OERs y la portabilidad de éstos entre distintos ROERs (Gértrudix-Barrio et al. 2007).

Es posible implementar un modelo pedagógico constructivista mediante el uso de recursos educativos abiertos (McCormick)

Los metadatos asociados a los objetos de aprendizaje deben describir el tipo de material, área del conocimiento, nivel o grado de los estudiantes para los cuales fue diseñado, objetivos de aprendizaje, idioma y requisitos técnicos para su uso.

De acuerdo con Hylén (2008) uno de los efectos positivos de compartir abiertamente los recursos pedagógicos es que el intercambio libre fomenta la difusión del conocimiento de forma más amplia y más rápida. Se potencia el desarrollo científico y técnico, y se descentraliza el conocimiento, lo que disminuye las desigualdades. Según McCormick (2004) es posible implementar un modelo pedagógico construc- tivista mediante el uso de recursos educativos abiertos.

\section{Conclusiones y retos de futuro}

Los repositorios de recursos educativos abiertos (OERs) permiten difundir el conocimiento en abierto para todos. Según la Unesco (2002) el aprendizaje abierto y a distancia es una fuerza que contribuye claramente al desarrollo social y económico, y actualmente es uno de los campos de la educación con mayor crecimiento.

El intercambio de OERs entre el profesorado permite mejorar la educación. Los repositorios de OERs (ROERs) han alimentado la cultura del compartir conocimientos, tanto a nivel individual como colectivo dentro de las instituciones de educación superior, cambiando las perspectivas del cuerpo docente sobre la propiedad intelectual de los materiales didácticos, lo que ha impulsado a su vez el nacimiento de una cultura nueva en la que se entiende que, cuando crece el capital intelectual, crece el capital humano, permitiendo y facilitando el desarrollo social.

Existe ambigüedad conceptual sobre la propiedad intelectual de los recursos educativos, ya que muchas universidades retienen los derechos de autor sobre lo que sus académicos producen y por lo tanto, ellos no tienen el derecho legal de compartir sus materiales libremente en la Red. Sin embargo, Ios ROERs facilitan los intercambios al disponer de licencias Creative commons para los objetos de aprendizaje que se depositan en ellos, lo que permite la cooperación académica.

Las condiciones tecnológicas actuales pueden consolidar la existencia e implementación de ROERs, ya que el software libre se puede instalar a un coste relativamente bajo. Además, considerando que la sociedad actual comprende mejor la democratización del conocimiento, unido al hecho de que las redes sociales fomentan compartir información, el desarrollo de una cultura de aprendizaje colectivo toma más fuerza.

La participación social en el acceso a la información y la elaboración de conocimientos, ha incrementado la necesidad actual en las universidades de replantearse críticamente las formas en las que se gestionan los contenidos y materiales de aprendizaje. Se evoluciona hacia la democratización del conocimiento y la información mediante la participación colectiva de las comunidades de enseñanza, lo que permite aprovechar la diversidad cultural de los recursos de aprendizaje y la capacidad intelectual de quienes los generan.

Si bien es difícil emular proyectos como el MIT OpenCourseWare (EUA) o el citado Xpert debido a la gran financiación que ambos reciben, son un modelo a observar para disponer de pautas de funcionamiento. Seguramente tendrán una gran influencia en las nuevas plataformas de apoyo a 


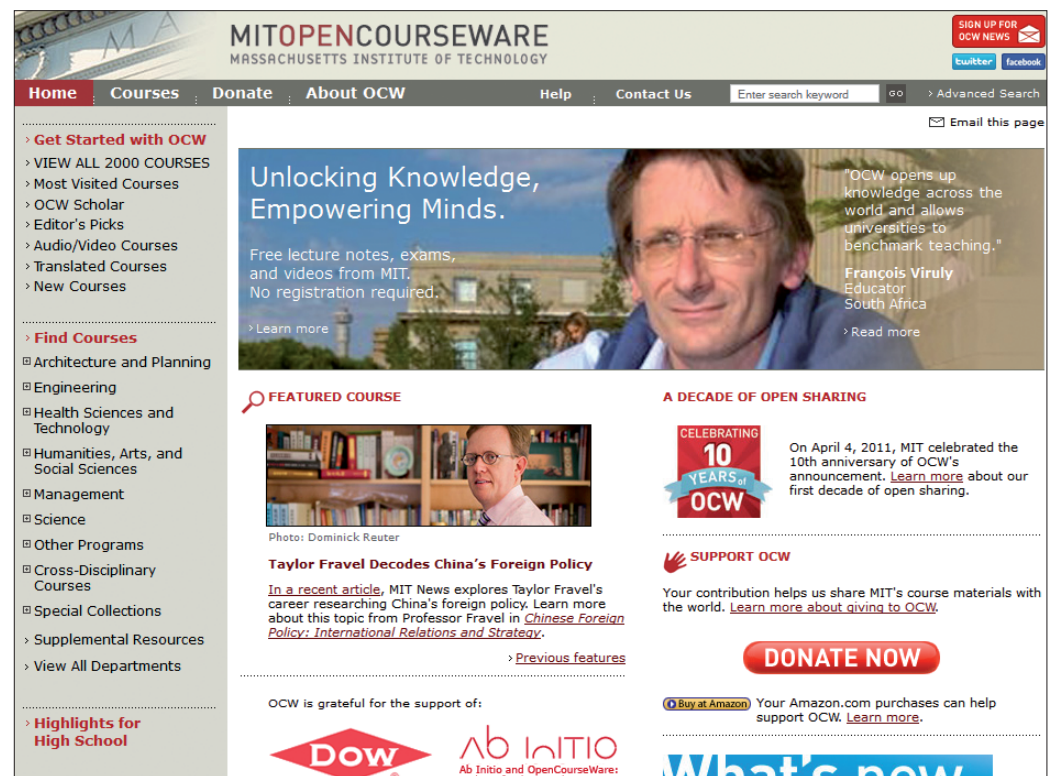

http://ocw.mit.edu/index.htm

las comunidades académicas y de mejora de la educación pública.

http://ocw.mit.edu/index.htm

Siguiendo las líneas propuestas por la declaración de Cape Town sobre educación abierta (2007), es necesario alentar a los docentes y estudiantes a crear y reutilizar recursos abiertos, y así animar la participación activa en el movimiento de educación democrática.

\section{Bibliografía}

Carson, Stephen. MIT OpenCourseWare 2005 Program evaluation findings report.

http://ocw.mit.edu/ans7870/global/05_Prog_Eval_Report_ Final.pdf

Ctoed. Cape Town open education declaration: unlocking the promise of open educational resources, 2008.

http://www.capetowndeclaration.org/read-the-declaration

Daniel, John; Uvalic-Trumbic, Stamenka. Commonwealth of learning: welcome \& introductory remarks, 2010.

http://www.col.org/resources/speeches/2010presentation/ Pages/2010-04-28.aspx

Downes, Stephen. "Models for sustainable open educational resources". Interdisciplinary journal of knowledge and learning objects, 2007, v. 3, pp. 30-44.

http://ijello.org/Volume3/IJKLOv3p029-044Downes.pdf

Gértrudix-Barrio, Manuel; Álvarez-García, Sergio; Galisteo-Del-Valle, Antonio; Gálvez-De-la-Cuesta, María-delCarmen; Gértrudix-Barrio, Felipe. "Acciones de diseño y desarrollo de objetos educativos digitales: programas institucionales". RUSC (Revista de universidad y sociedad del conocimiento), 2007, v. 4, n. 1, pp. 14-25.

http://rusc.uoc.edu/ojs/index.php/rusc/article/view/v4n1gertrudix-alvarez-galisteo-galvez-gertrudix

Gurell, Seth; Kuo, Yu-Chun; Walker, Andrew. "The pedagogical enhancement of open education: an examination of problem-based learning". The intl review of research in open and distance learning, 2010, v. 11, n. 3, pp. 95-105. http://www.irrodl.org/index.php/irrodl/ article/view/886/1633

Hylén, Jan. Open educational resources: opportunities and challenges. Paris: OECD's Centre for Educational Research and Innovation, 2008.

http://www.knowledgeall.net/files/ Additional_Readings-Consolidated.pdf

Longmire, Warren. "A primer on learning objects". San Francisco: American Society for Training \& Development, 2000.

http://www.astd.org/LC/2000/0300_longmire.htm

Margaryan, Anoush; Milligan, Colin; Douglas, Peter; Littlejohn, Allison; Nicol, David. Deliverable 10 recommendations to JISC for future research and development: community dimensions of learning object repositories. London: JISC, 2007.

http://ie-repository.jisc.ac.uk/107

McCormick, Robert. "Issues of learning and knowledge in technology education". Intl journal of technology and design education, 2004, v. 14, n. 1, pp. 21-44.

http://oro.open.ac.uk/818/

http://dx.doi.org/doi/10.1023/B:ITDE.0000007359. $81781.7 c$

McCracken, Richard. "Cultural responses to open licences and the accessibility and usability of open educational resources". En: Expert meeting on Open Educational Resources, Malmö, 2006.

http://www.oecd.org/dataoecd/48/38/36539322.pdf

McGreal, Rory. "Online education using learning objects". Routledge Psychology Press, Falmer, 2004, ISBN: 9780415335126

OECD. Giving knowledge for free: the emergence of open educational resources. Paris: Centre for Educational Research and Innovation, 2007. ISBN: 9789264031746 http://www.oecd.org/dataoecd/35/7/38654317.pdf

Rodríguez-Morales, Germania; Cueva-Carrión, Samanta. "OER, estándares y tendencias". RUSC. Revista de universidad y sociedad del conocimiento, 2010, v. 7, n. 1.

http://rusc.uoc.edu/ojs/index.php/rusc/article/view/v7n1_ cueva_rodriguez

Siemens, George. "Why we should share learning resources". Elearnspace, 29 mayo 2003.

http://www.elearnspace.org/Articles/why_we_should_ share.htm

Unesco. Forum on the impact of open courseware for higher education in developing countries, 2002.

http://portal.unesco.org/ci/en/files/2492/1033056740 4OCW_forum_report_final_draft.doc/OCW_forum_report_ final_draft.doc

Unesco. A basic guide to Open Educational Resources (OER), Vancouver: Commonwealth of Learning, 2011. ISBN: 9781894975414

http://oer.unescochair-ou.nl/?wpfb_dl=29 